\title{
Popper and Bohr on Realism in Quantum Mechanics
}

\author{
Don Howard \\ Department of Philosophy, University of Notre Dame, Notre Dame, Indiana, United States. E-mail:dhoward1@nd.edu
}

Editors: Danko Georgiev \& Peter J. Lewis

Article history: Submitted on June 18, 2012; Accepted on November 1, 2012; Published on November 15, 2012.

\section{$P$}

opper's program in the foundations of quantum mechanics defending objectivity and realism developed out of a profound dissatisfaction with the point of view associated with Bohr, which is usually designated the Copenhagen interpretation. Here I will argue that while Popper's aim is a noble one, his program does not succeed on two counts: he does not succeed in showing that Bohr's philosophy must be rejected as a variety of subjectivism, and his alternative interpretation of indeterminacy rests on a highly questionable assumption according to which simultaneously precise conjugate parameters are possible. Nevertheless I like Popper's propensity interpretation of probability and think that the propensity idea deserves further research.

Quanta 2012; 1: 33-57.

\section{Introduction}

For years I have been puzzled, by the attitudes of my colleagues in philosophy and physics toward Popper's work in the foundations of quantum mechanics. His program developed out of a profound dissatisfaction with the point of view associated with Bohr, which is usually designated the Copenhagen interpretation. He has severely criticized Bohr's philosophy, calling it the "ruling dogma" [1, p. 7], and he presents his own interpretation of the quantum

(c) (1) This is an open access article distributed under the terms of the Creative Commons Attribution License CC-BY-3.0, which permits unrestricted use, distribution, and reproduction in any medium, provided the original author and source are credited. theory as an explicit alternative. And yet, with the exception of one essay by Paul Feyerabend [2,3], no one who professes sympathy with Bohr's interpretation has troubled to respond, in a systematic way, to Popper's criticism, nor have they undertaken a comprehensive critique of Popper's views. It may be that Bohr's sympathizers view Popper's work as unworthy of a reply. But my guess is that their reticence is to be explained not so much by arrogance, as by a disheartening sense of their own failure to have understood fully what Bohr intended. In any case, the silence is embarrassing; a reckoning is in order.

Popper's program in the foundations of quantum mechanics is continuous with his efforts elsewhere to defend objectivity and realism. It is an essential part of his larger philosophical enterprise because the apparent novelties of the quantum theoretical description of nature have, on occasion, been called upon to give scientific authority to philosophical attacks on realism and objectivity. The defense is two-pronged. It includes, on the one hand, a critical analysis of the subjectivism which allegedly infects the Copenhagen interpretation, and, on the other hand, provision of objective interpretations of both indeterminacy and quantum mechanical probabilities. I will argue that while Popper's aim is a noble one, his program does not succeed on two counts: he does not succeed in showing that Bohr's philosophy must be rejected as a variety of subjectivism, and his alternative interpretation of indeterminacy rests on a highly questionable assumption. But I like his interpretation of probability, the propensity interpretation. I think the propensity idea is Popper's one genuine contribution to the foundations of quantum mechanics, and its further development ought to be one of 
the principal aims of current research. But I also believe that when the propensity interpretation is divorced from the remainder of Popper's program, it no longer looks like an alternative to Bohr's position; it appears, instead, to confirm some of the latter's basic insights.

\section{Realism, objectivity and the interpretation of quantum mechanics}

In 1927, Popper was finishing his Ph.D. at the University of Vienna when two important ideas made their appearance. The first was Heisenberg's uncertainty or indeterminacy principle [4]; the second was Bohr's complementarity interpretation of quantum mechanics [5-7]. The exact meaning of each is to this day the subject of considerable disagreement, but to a first degree of approximation they can be characterized as follows.

From a formal point of view, the indeterminacy principle asserts the existence of an upper bound on the product of the standard deviations of any two conjugate parameters of a physical system. In the familiar case of position and momentum, this limitation is expressed by the well-known equation: $\Delta q_{x} \Delta p_{x} \geq \frac{\hbar}{2}$, where $\Delta q_{x}$ and $\Delta p_{x}$ represent, respectively, the indeterminacies of the $x$-components of the position and momentum of a system. But while the derivation of this equation and its analogues for other pairs of conjugate coordinates is a fairly straightforward matter (in the case of the energy-time indeterminacy relation, even the derivation is a matter of dispute; the basic difficulty concerns the possibility of representing the time by a Hermitian operator) [8, pp. 136-156], their interpretation has been a topic of controversy, various thinkers having argued (and this is by no means an exhaustive catalogue of the different points of view) that the indeterminacies represent (1) measures of an intrinsic indefiniteness of the coordinates themselves, (2) measures of the precision with which individual measurements of the coordinates can be carried out, or (3) measures of the spread of results obtained in a series of measurements, each of which may, itself, be as precise as desired.

Bohr's interpretation of quantum mechanics shares with the indeterminacy principle a concern with the apparently novel relationship between conjugate coordinates. Bohr argues that the kinds of experimental arrangements suited to the measurement of conjugate coordinates are invariably mutually exclusive, in the sense that, for example, a position measurement can never be performed with exactly the same apparatus, nor even in the presence of the same apparatus, which we use for a momentum measurement [9, p. 699]. Since he also believes that, in addition to their role in measurement, experimental arrangements are somehow crucial in the very definition of the properties of quantum objects (this is not merely a variety of operationism; for Bohr the experimental conditions are necessary, but not sufficient, conditions for definition) [9, p. 700], Bohr concludes:

\section{Consequently, evidence obtained under differ- ent experimental conditions cannot be compre- hended within a single picture, but must be regarded as complementary in the sense that only the totality of the phenomena exhausts the possible information about the objects. [10, p. 210]}

Philosophers, and even many physicists, were quick to attempt to extract philosophical lessons from quantum mechanics. In the very lecture in which he introduced complementarity, Bohr comments:
Now, the quantum postulate implies that any observation of atomic phenomena will involve an interaction with the agency of observation not to be neglected. Accordingly, an indepen- dent reality in the ordinary physical sense can neither be ascribed to the phenomena nor to the agencies of observation. [7, p. 54]

Reading this and many similar pronouncements, Popper thought he detected a common theme, which he took to be characteristic of the views thought to be shared by Bohr and his associates at Copenhagen:

\section{... the Copenhagen interpretation of quantum mechanics ... says that "objective reality has evaporated" and that quantum mechanics does not represent particles, but rather our knowl- edge, our observations, or our consciousness, of particles. [1, p. 7]}

Later I will question the assumption that there is a single Copenhagen interpretation, for there are important differences between Bohr and even his close colleagues, like Heisenberg. But the contrary impression is widespread, so it is not surprising that Popper adopts it uncritically (Heisenberg, himself, is largely responsible for the confusion [11]. He freely uses the label Copenhagen interpretation to denote both his views and Bohrs [12]). In any case, the threat which such a viewpoint poses to Popper's realism is substantial, for it suggests that realism is contradicted by a physical theory with impeccable empirical credentials. Moreover, a number of thinkers who allied themselves with the Vienna positivists were quick to draw just such a conclusion. The confluence of both philosophical and scientific doubts about the existence of 
a real external world surely made the case against realism look formidable.

Popper's arguments against positivism are familiar enough not to need repeating here. But his arguments against drawing idealist or subjectivist conclusions on the basis of quantum mechanics are far less well known, in spite of the importance which Popper himself attaches to them.

According to Popper, realism and objectivity are not contradicted by the formulae of quantum mechanics, the correctness by of which he does not question, but only by a mistaken interpretation of these formulae [13, §75]. And the primary reason for this mistaken interpretation, so he claims, is a failure to understand correctly the nature of quantum mechanical probability statements [14, p. 73] [1, p. 28]:

\section{... the problems of the interpretation of quan- tum mechanics can all be traced to problems of the interpretation of the calculus of probabil- ity. [15, p. 104]}

The quantum mechanical formalism describes a physical system by means of a mathematical device called a state function (commonly referred to as the $\psi$ function), a different state function being assigned to the system for every different state that it can occupy (hence one typically speaks indifferently of states and state functions). Various purely mathematical manipulations of the state function enable one to derive predictions concerning the outcome of measurements that can be performed on the system. The Heisenberg indeterminacy formulae are themselves the result of such manipulations. The fact that the $\psi$ function arises as a solution of the Schrödinger equation (the fundamental dynamical equation of quantum mechanics), which bears a formal analogy to the classical wave equation, led Schrödinger and other nonCopenhagen quantum theorists to believe that electrons and other elementary particles in some sense really are waves [8, pp. 24-33] [16] [17, pp. 43-80]. But ever since Born's pioneering statistical interpretation of the $\psi$ function, there has been widespread agreement that the $\psi$ function is best viewed as providing a measure of the probability of a system's being found, upon measurement, to have a specific value of a given variable [18] [8, pp. 38-44].

While agreeing that quantum mechanics is a probabilistic theory, Popper at first denied that this fact alone proves that nature itself is not deterministic. In the 1930s he argued that the determinism issue was essentially a metaphysical question, and hence not scientific, but he betrayed a certain sympathy for determinism, at least in the form of a heuristic maxim to the effect that scientists should always search for strict laws [13, §78]. Later, however, he came to regard the determinism thesis as a testable hypothesis, and expressed his preference for a type of objective indeterminism [19,20]. In this respect, Popper stands alone among the realist critics of Bohr and Heisenberg, many of whom, including Einstein have held the opinion that realism and determinism go hand in hand. In a letter to Born, Einstein says:

I admit, of course, that there is a considerable amount of validity in the statistical approach ... I cannot seriously believe in it, because the theory cannot be reconciled with the idea that physics should represent a reality in time and space, free from spooky actions at a distance. [21, p. 158]

Instead of focusing on the determinism issue, Popper's dispute with Bohr and Heisenberg concerns the meaning of the probability statements which quantum mechanics necessarily employs. In Popper's account, the Copenhagen view traces the need for probabilities to quantum mechanical limitations on our knowledge of the properties of atomic systems, and thus ascribes a subjective interpretation to quantum mechanical probabilities. Popper, on the other hand, traces the probabilistic character of quantum mechanics to the statistical character of its problems, and would thus give an objective interpretation to its probability statements. In the 1930 s this meant for him a classical frequency interpretation, later it meant a propensity interpretation. What exactly is meant by the various kinds of interpretations will be considered in some detail below.

The distinction between objective and subjective interpretations is crucial, for Popper holds that all of the objectionable consequences of the Copenhagen interpretation, including its denial of realism, stem from its predilection for a subjective interpretation of probability statements. In his own words:

... it is this mistaken belief that we have to explain the probabilistic character of quantum theory by our (allegedly necessary) lack of knowledge, rather than by the statistical character of our problems, which has led to the intrusion of the observer, or the subject into quantum theory. [1, p. 17]

If this is the source of the problem, then to defend realism and objectivity from the quantum mechanical challenge, one need only establish the tenability of an objective interpretation of quantum me chanical probabilities. This is precisely Popper's strategy. 


\section{Indeterminacy and subjectivism}

The topic of Popper's first disagreement with the Copenhagen theorists was the interpretation of the Heisenberg indeterminacy formulae; it was here that Popper thought he detected most clearly the Copenhagen penchant for subjectivism. But while indeterminacy was the immediate focus of Popper's initial critique, the implications of that critique were widespread, touching already upon the central problem of the meaning of the quantum mechanical state function. Since the supposed need to combat the malaise of subjectivism is what gives meaning and purpose to Popper's whole program in the philosophy of quantum mechanics, we would do well to examine this part of his argument in considerable detail.

From the start, the discussion of indeterminacy, even among those identified as the members of the Copenhagen school, was characterized by a tension between at least two different interpretations. In some cases one even finds a vacillation between the two in the work of an individual physicist. The first takes indeterminacy to be merely a limitation on measurement, while the second takes it to be a reflection of some intrinsic indefiniteness of the coordinates of atomic systems. Both views entail a limitation on how precisely we can know a system's coordinates.

Heisenberg, himself, tended toward the former interpretation, though he was by no means wholly consistent in his published pronouncements on the subject. Typically, he illustrated the indeterminacy relations with thought experiments which purport to show how a measurement of an electron's position, following close up on a momentum measurement, would necessarily interfere with or disturb the electron's momentum in an inherently unpredictable manner, so that the prior momentum measurement would be rendered useless for the purposes of prediction [22, pp. 21-30]. This means that the physical disturbance caused by the position measurement necessarily renders imprecise our previously obtained knowledge of the electron's momentum, and, because it thus focuses our attention on limitations on knowledge, Poper labels this reading of indeterminacy subjective [13, p. 215].

Following the lead of Schrödinger's wave mechanics, a number of other physicists opted for the intrinsic indefiniteness interpretation of indeterminacy. According to this view, the Heisenberg formulae imply that it is physically impossible for a system like an electron even to possess simultaneously definite values of any two conjugate variables The unavoidable imprecision in our knowledge of the value of these coordinates is thus explained as the reflection of a deeper-lying indefiniteness of the coordinates themselves. Because it regards indeterminacy as a property of the system itself, and not directly as a function of our limited knowledge of what might actually be a well-defined system, Popper terms this approach objective [13, p. 215].

At the time he published Logik der Forschung (1935), Popper had had no formal advanced training in physics he says of his abilities in this area: "I felt in the end that I was not really good enough" [15, p. 57] and yet he already sensed the physicists' confusion over indeterminacy more clearly than did many physicists themselves. In an interesting analysis, Popper traces the vacillation between the subjective and objective approaches to indeterminacy to the physicists' desire to reconcile what, at the time, Popper took to be ultimately inconsistent features of the quantum theory as it was then understood [13, pp. 210211]. On the one hand, the apparent success of quantum mechanics in explaining such fundamental phenomena as the stimulated emission of radiation from atoms, the photoelectric effect, and the specific heats of solids (all of which were embarrassing problems for classical theories of radiation and matter), together with the resolution of doubts about the strict conservation of energy in individual atomic processes, encouraged physicists to view quantum mechanics as a fundamental theory of the mechanics of individual systems. Such a view also accorded with a traditional conception of physics as the basic science of nature, the science which concerns itself with the deepest level of structure. According to Popper, it was this attitude which initially inclined physicists toward the objective approach. At the same time, however, the emerging consensus, especially among those termed the Copenhagen theorists, was that quantum mechanics is an irreducibly probabilistic theory, and this belief had just received impressive reinforcement at the time Logik der Forschung was being written, in the form of von Neumann's famous, though, as we now know, mistaken, proof of the impossibility of deterministic, hidden variable interpretations of quantum mechanics [23, pp. 313-325] [24]. The problem, Popper suggests, was in understanding how quantum mechanics could be both a theory of individual systems and a probabilistic theory. In Popper's account, it was the physicists' striving to resolve this difficulty that led them down the garden path to subjectivism.

On the face of it, there is no difficulty; the objective approach to indeterminacy seems to hold the key. One could simply argue that theory's probabilistic character stemmed from an intrinsic indefiniteness in the states of individual systems, or, more specifically, from an indefiniteness in some or all of the parameters, such as position and momentum, which go to make up the state; the uncertainties mentioned in the Heisenberg formulae would be the measures of this indefiniteness. Under these circumstances, probabilistic predictions would be the norm, for if, say, the position of a system is intrinsically indef- 
inite at a given time, then no matter how deterministic the theory's dynamical laws are, they can at best yield only a range of possible positions for the system at any subsequent time. But however attractive this analysis might initially appear, certain considerations raise serious doubts about its cogency, doubts which go far beyond the mere offense to our classical intuitions, according to which indeterminacy is always but an appearance, masking a still deeper deterministic reality.

From a physical point of view, the most vexing difficulty concerns the famous problem of the collapse or reduction of the wave packet [13, pp. 231-232] [1, pp. 34-38]. If we are to regard indefiniteness as an intrinsic property of an individual system, then we must regard the state function as a description of the intrinsic state of that system (as opposed to regarding it as a representation of our knowledge of the system). In the most general case, which is typical of a system prior to the performance of a measurement on it, the state function is of a type known as a superposition, a weighted, complex sum of a number of different state functions, each of which, by itself, corresponds to a state wherein the system has a definite value of the observable to be measured. At the very least, a system described by a superposition cannot be known, with certainty, to be in any one of the component states, called the eigenstates of the observable; but one can calculate the probability of the system's being found, upon measurement, to have any specific value of the observable. Repeated measurements on identically prepared systems will yield different results, but the distribution of those results will conform to the predicted probabilities. Whether the system can be said actually to $b e$ in one of the eigenstates prior to the measurement is an important question of interpretation. (It is the presence of superpositions in the formalism that gives rise to non-zero indeterminacies. The indeterminacy principle's restriction on conjugate parameters is related to the formalism's not admitting simultaneous eigenstates of conjugate observables. If a system is in an eigenstate of one observable, it will be in a superposition with respect to any conjugate observable.)

Being committed to the view that the state function describes the state of the system itself, the proponents of intrinsic indefiniteness must conceive of the state corresponding to a superposition as somehow "blurred" or "smeared". They would have to deny that a system thus described is in any definite eigenstate, be it known or unknown. The problem with this view is that measurement always yields a definite result, and if the measurement is a non-destructive one (for example, a filter that only allows systems with a definite value of the observable to pass), then immediately after the measurement a system is always describable by a state function corresponding to one of the eigenstates represented in the original superposition. Such a description of the post-measurement state of the system is correct, in the sense that it leads to the proper predictions of the system's subsequent behavior. It would appear then, from the objective point of view, as though a system originally in a "smeared", indefinite state collapses upon measurement to some definite state.

But - and this is why the collapse is a problem - quantum mechanics has no way of explaining the collapse. If the state function is taken to describe a system's intrinsic properties, then it is hard to avoid the conclusion that the collapse is a real physical phenomenon, but the dynamics of the quantum theory make no provision for such a quasi-instantaneous, discontinuous transition; certainly the Schrödinger equation, the basic dynamical equation of quantum mechanics, cannot account for such a process. The best the proponents of the objective approach can do in the face of this difficulty is to follow von Neumann in taking the collapse as an independent postulate (the so-called projection postulate) [23, pp. 347-358] [8, pp. 226-230]. This strategy is not objectionable from an empirical point of view, but the ad hoc character of the postulate makes its presence an aesthetic defect in an otherwise elegant theory.

Popper was neither the first nor the only commentator to draw attention to the troubling implications of the problem of wave packet collapse. But over the years he voiced other criticisms of the idea that a superposition describes the intrinsic state of an individual system. One of the most important is his contention that from the point of view of the objective indefiniteness interpretation, the quantum formalism contains metaphysical elements [13, p. 215]. The details of the pertinent arguments will be reviewed and criticized in section 4 , but briefly, what Popper maintains is that while precise predictions of all aspects of the future behavior of quantum systems may be impossible, completely precise calculations of all aspects of the past behavior of quantum systems are possible according to the formalism. If, as the objective indefiniteness interpretation asserts, quantum systems never posses simultaneously precise values of all coordinates, then the results of these calculations are, to say the least, anomalous.

Among the alternatives open to those who are dissatisfied with the objective indefiniteness interpretation of the state function and the Heisenberg formulae, two are prominent in Popper's deliberations. The one favored by Popper continues to regard quantum mechanics as a probabilistic theory, but denies that it is itself a fundamental theory of individual systems, viewing the state function instead as a description of the statistical properties of an ensemble of systems, and the Heisenberg formulae as what Popper calls statistical scatter relations. This view assumes that individual systems do always possess 
simultaneously definite values of all their coordinates, an ensemble of systems displaying the appropriate scatter in these values. The other alternative is to continue to regard quantum mechanics as a probabilistic theory of individual systems, but to construe the state function as describing not the intrinsic physical properties of microsystems, but, in some way, our knowledge of these properties. This view is of a piece with the limitations-on-measurement interpretation of indeterminacy, if the latter is construed, following Popper, as a statement about limitations on the possible extent of our knowledge of atomic systems; and like the limitations-on-measurement idea, it earns from Popper the designation subjective [13, p. 215]. However, to avoid begging any questions, I will refer to it as the epistemic interpretation of the state function.

This interpretation has its advantages. For instance, to say that a superposition correctly describes a system is merely to say, on this approach, that our knowledge of the system is limited in such a way that we know only the likelihood of one of its variables being found to have a certain value; no claim about the intrinsic state of the system is entailed, and the resulting difficulties of the objective interpretation are avoided. In particular, the collapse of the state function is regarded, from this perspective, simply as a sudden, discontinuous change in the degree of precision of our knowledge of the system, something which is not at all surprising, given that the aim of measurement is to enhance our knowledge of the object of measurement. Another advantage is that the epistemic interpretation enables us to continue regarding quantum mechanics as a theory of individual systems, at least in the sense that the knowledge which it takes as fundamental can be viewed as knowledge, however imprecise, of individual systems.

Problems such as the collapse of the wave packet were a common topic of discussion among the early developers of the quantum theory, and undoubtedly were crucial in leading some to favor the epistemic interpretation of the state function. A general sympathy with positivist scruples regarding talk of unobservable entities should also not be discounted as an influence on their thinking. But Popper has suggested that the situation in the foundations of probability was among the most important factors favoring this choice. He notes that in the late 1920s and early 1930s, crucial years in the development of the Copenhagen interpretation, the only objective theory of probability under serious discussion was the relative frequency interpretation, which defines the probability of one kind of event, called an outcome, as its frequency, relative to other outcomes, in an infinite sequence of other events, called trials. According to Popper, this definition presents a problem for those who attempt to construe indeterminacy and quantum mechanical probabilities gen- erally as objective properties of individual quantum systems. The difficulty is that, as it stands, the frequency theory does not permit the unambiguous ascription of probabilities to individual events, such as getting a six on a specific throw of a die; one could, in principle, have different probabilities for that event, depending on which infinite sequence of trials it is included in. But this means that the attribution of an objective probability, in the relative frequency sense, to a specific event is meaningful only relative to a specification of an infinite sequence of trials, which is really to say that such a probability is a property not of the individual event, but of the infinite sequence itself. Since the interpretation of quantum mechanics as a fundamental theory of individual systems seems to require the attribution of probabilities to individual events (such as the passage of a specific photon through a filter), these probabilities cannot, therefore, be understood as relative frequencies. And if the relative frequency interpretation is the only objective interpretation of probability, it follows that individual quantum mechanical probabilities are not objective [13, §71] [15, §34].

In the period under discussion, the principal alternative to the frequency interpretation was one which derives the probability function from a measure of a person's degree of confidence in the truth of a proposition. This is commonly referred to as the subjective interpretation of probability. It poses no obstacle to our regarding probabilities as properties of individual events and systems, as long as it is understood that the connection with individual events is indirect, in the sense that probabilities, subjectively construed, are, strictly speaking, measures of one's faith in the truth of propositions describing the events, and only secondarily properties of the events and systems themselves. In particular, subjective probabilities are not infected with the same kind of ambiguity which plagues probabilities regarded as relative frequencies; different individuals might assign different probabilities to one and the same proposition (this is, of course, a large part of what is meant by calling such probabilities subjective, and a major reason why Popper opposes this interpretation), but there is no systematic obstacle to any one individual's assigning a unique probability to a proposition describing a single event. (For Popper's objections to the subjective interpretation of probability, see [25] [13, §48 and $\S 62])$. It is precisely because the subjective interpretation gives us a way to speak of probabilities as properties of individual events and systems that Popper identifies it as the primary motivation for many physicists' having interpreted quantum mechanical probabilities, and thus the state function, in an epistemic, or, in Popper's words, subjective, fashion. He says:

Now frequency theorists hold that there are ob- 
jective questions concerning mass phenomena, and corresponding objective answers. But they have to admit that whenever we speak of the probability of a single event, qua element of a mass phenomenon, the objectivity becomes problematic; so that it may well be asserted that with respect to single events, such as the emission of one photon, probabilities merely evaluate our ignorance. For the objective probability tells us only what happens on the average if this sort of event is repeated many times: about the single, event itself the objective statistical probability says nothing. It was here that subjectivism entered quantum mechanics ... [15, p. 178]

Popper marshals evidence, in the form of quotations from a variety of authors, in order to convince us that, from the time of the consolidation of the modern quantum theory in the late 1920s,

physics had become a stronghold of subjectivist philosophy, and it has remained so ever since. [15, p. 177]

We read from Moritz Schlick, one of the fathers of Vienna positivism:

Of natural events themselves it is impossible to assert meaningfully any such things as "haziness" or "inaccuracy". It is only to our own thoughts that anything of this sort can apply... [13. pp. 215-216] [26]

Sir James Jeans is found commending a similar view:

In brief, the particle picture tells us that our knowledge of an electron is indeterminate; the wave picture that the electron itself is indeterminate, ... Yet the content of the uncertainty principle must be exactly the same in the two cases. There is only one way of making it so: we must suppose that the wave picture [the $\psi$ function is intended here] provides a representation not of objective nature, but only of our knowledge of nature ... [13, p. 229] [27, p. 235]

Popper comments on the preceding quotation:

Schrödinger's waves are thus for Jeans subjective probability waves, waves of our knowledge. And with this, the whole subjectivist probability theory invades the realm of physics. [13, p. 229]
The list of citations could easily be extended; there is no denying that quantum mechanics has frequently been construed as referring to our knowledge, rather than to the world, especially by thinkers either belonging to or influenced by the tradition of Vienna positivism. To give just one more example, consider the following more recent remark by Heisenberg:

The conception of the objective reality of the elementary particles has thus evaporated in a curious way, not into the fog of some new, obscure, or not yet understood reality concept, but into the transparent clarity of a mathematics that represents no longer the behavior of the elementary particles but rather our knowledge of this behavior. [28, p. 100]

But Popper wants to claim much more than this. He wants to claim, first, that the dominant interpretive tradition since the 1930s - such is the stature, in his estimation, of the Copenhagen interpretation - is subjectivistic in import and intent, second, that the allegedly subjectivistic Copenhagen interpretation is the result of a mistaken subjective interpretation of quantum mechanical probabilities; and third, that this "intrusion of the subject" into quantum theory leads the Copenhagen theorists to conclude that "objective reality has evaporated". About each of these claims serious questions must be raised. In particular, as I mentioned earlier, I want to criticize the assumption that the opinions of Bohr and Heisenberg coalesce into a single interpretation. A subjectivist epidemic may have raged in Copenhagen, but some people might not have succumbed.

First we must pause to consider carefully what Popper means when he labels a point of view subjective. That an unflattering contrast with objective is intended is clear. He remarks, for example:

... if one interprets the Heisenberg formulae (directly) in a subjective sense, then the position of physics as an objective science is imperilled. [13, p. 229]

But the meaning of subjective is not exhausted by this contrast. We have found that what earns an interpretation the designation subjective from Popper is its being somehow concerned with our knowledge of physical systems, rather than with the systems themselves. But if subjective interpretations of quantum mechanics are to be opposed because they represent a threat to objectivity, there is something puzzling about simply making reference to knowledge the criterion of subjectivity, for as Popper himself has stressed in other contexts, there are both subjective and objective types of knowledge: 
Now I wish to distinguish between two kinds of 'knowledge': subjective knowledge (which should better be called organismic knowledge, since it consists of the dispositions of organisms); and objective knowledge, or knowledge in the objective sense which consists of the logical content of our theories, conjectures, guesses ... [29, p. 73]

In terms of Popper's later "three worlds" ontology, subjective knowledge is knowledge in its World 2 aspect, where World 2 is "the mental world, or world of mental states" [29. p. 154] (in Popper's philosophy, there is no inconsistency in regarding subjective knowledge as both a mental state and a disposition of an organism. He rejects a Cartesian dualism of mental and physical substances, but advocates a dualism of mental and physical states. A mental state is, thus, for Popper, just a special kind of dispositional state of an organism [29, p. 231]), and objective knowledge is knowledge in its World 3 aspect, this world being characterized as:

... the world of intelligibles, or of ideas in the objective sense; it is the world of possible objects of thought: the world of theories in themselves, and their logical relations; of arguments in themselves; and of problem situations in themselves. [29, p. 154]

It is the existence of just this ambiguity in our talk of knowledge which led me to speak above of epistemic interpretations, rather than subjective ones, the point being that talk of knowledge may not be, automatically, a sign of subjectivism.

In view of the prominent role which Popper assigns to the objective/subjective knowledge distinction, one would assume that when he indicts an interpretation as subjectivist because it makes essential reference to knowledge, he means to indict it for referring to knowledge in the subjective sense, but while his bill of particulars usually includes the expression subjective knowledge or one of its cognates [29, p. 141], he does not always specify the nature of the crime this carefully. Popper no doubt wants to criticize reference to either kind of knowledge in any interpretation of a physical theory which makes our knowledge, rather than the world, the focus of the theory's attention, for the claim that theories tell us about our knowledge, rather than the world, does not harmonize with a realist attitude. Moreover, one of the traditional (though, I would argue, misleading) uses of the terms subjective and objective is precisely to mark the knowledge/world distinction. So while it may be confusing, the distinction between subjective and objective knowledge might simply be irrelevant to Popper's discussion of epistemic interpretations of quantum mechanics.
But I do not think that this is the case. Popper was too systematic a philosopher not to have noted such a confusion and sought to correct it. My suggestion is that he believed that subjectivism is the same sin, wherever it might be encountered, and, thus, that the subjective character of an interpretation of quantum mechanics consists in nothing more and nothing less than its taking the theory to tell us about our subjective knowledge of events, knowledge in the World 2 sense, rather than the events themselves. In a later essay he said:

...it is not surprising that neglect of the third world - and consequently a subjectivist epistemology - should be still widespread in contemporary thought. Even where there is no connection with Brouwerian mathematics there are often subjectivist tendencies to be found within the various specialisms, I will here refer to some such tendencies in logic, probability theory, and physical science. [29, p. 140]

The story which he then sketches about subjectivism in probability theory and physics is the same one we have been reviewing in this section. It is only in his early discussion of subjectivism in quantum mechanics, in The Logic of Scientific Discovery, that Popper fails to heed the distinction between subjective and objective knowledge, but in that discussion the same work is performed by a distinction which prefigures the later one, namely, a distinction between "our knowledge" simpliciter and "objective science", the former being defined, just as subjective knowledge is later defined, as a "system of dispositions". And about "knowledge", thus defined, Popper adds:
But all this interests only the psychologist. It does not even touch upon problems like those of the logical connections between scientific statements, which alone interest the epistemol- ogist. [13, p. 80]

Thus, whether Popper speaks just of "knowledge", in his early work, or more pointedly of subjective knowledge, in his later work, his charging an interpretation with subjectivism means simply that it reads quantum mechanics as telling us about a kind of knowledge which is the private possession of individual knowing subjects, rather than about the physical world.

There is a good reason for being careful to show that what motivates Popper's charge of subjectivism is a worry about reference to knowledge of the specifically subjective type. For this criticism, directed against Bohr, Heisenberg and the other Copenhagen theorists, is a crucial part of Popper's argument; it is what first establishes the need for an alternative, objective interpretation of quantum me- 
chanics, which Popper then provides. But I want to argue that the charge, thus understood, is unfounded.

As noted above, it is easy to accumulate a list of references to knowledge in the writings of commentators on quantum mechanics who are loosely associated with Bohr. Some of them, surely, can plausibly be read as references to subjective knowledge (Schlick's mention of "our own thoughts" is an example), but many, if not most, cannot (I would be reluctant to read Jeans talk of "our knowledge of nature" in this way). The fact is that many, often ill-considered things have been said about quantum mechanics by philosophers and, especially, by physicists. And it is pointless to press these remarks too hard with fine, philosophical distinctions. I would guess that if we explained the subjective/objective knowledge distinction to Jeans, he would say that, of course, he meant knowledge of the objective sort, but it is in the nature of the case that such conjectures cannot easily be established. It is a far better strategy for critic and defender simply and straight-forwardly to consider the facts. Thus, we must address the question as to whether or not there is anything about the quantum, theory that would lead someone to assign subjective knowledge a special place in its interpretation.

The hallmark of objective knowledge, according to Popper, is its susceptibility to inter-subjective testing and criticism [13, p. 25]. Is there any reason to suspect that our knowledge of quantum objects and events falls short of this standard? The original source of the worry over subjectivism in quantum mechanics is doubtless Heisenberg's unfortunate explanation of indeterminacy as the inevitable consequence of the observer's disturbance of the object of observation. Popper is quite explicit about this. He describes the Copenhagen interpretation as:

... the claim that, in atomic theory, we have to regard "the observer" or "the subject" as particularly important, because atomic theory takes its peculiar character largely from the interference of the subject or the observer (and his "measuring agencies") with the physical object under investigation. [1, p. 10]

We seem to be confronted here with a clear-cut failure of objectivity, since the magnitude of the disturbance allegedly occasioned by the observer's intervention presumably varies from observer to observer, and from observation to observation, owing to its inherent unpredictability (or "uncontrollability", in Bohr's quaint, early terminology) [22, p. 3] [7, pp. 57-68].

But it would be seriously misleading to describe this situation simply as a failure of objectivity, and to make it the basis for the charge that physics has become the stronghold of subjectivism. For one thing, we must be careful about just who or what does the disturbing. While Heisenberg himself sometimes speaks of the observer as the "subject", [22, p. 2] conjuring up the image of the denizens of World 2, the proper object of our concern is the purely physical interaction between the quantum system under investigation and the observer, the latter being considered now as nothing more than an especially complicated physical system. Indeed, it is better to speak simply of an interaction between the object and a measuring instrument, and to leave conscious human observers out of the picture entirely, for whatever the novelties of observation in quantum mechanics, they remain the same if human observers are replaced by instrumentation attached to automatic recording devices. Thus, even if it were true that every observation disturbs the observed object in a different way, the difference is not due to the involvement of different human subjects. It is, instead, a mundane physical fact of a purely objective, World 3 sort. Furthermore, factoring out the human observer makes it clear that the knowledge derived even from "disturbing" observations is totally objective, for the result preserved by the recording device needs only to be formulated as a statement in order to become a publicly debatable, World 3 entity, something that all researchers can agree upon. Some physicists would question the claim that the recorded results are objective. The most straightforward description of the measurement process implies that an instrument interacting with a system described by a superposition will itself be in a superposition at the conclusion of the measurement interaction, whereas I am assuming that the instrument winds up in a definite state. If it is in a superposition, then different observers might, conceivably, find it in different states when they look at it. Of course, I question this simple description of measurement, but, unfortunately, there is no consensus among physicists on an adequate quantum theory of measurement [8, pp. 471-521] [30, pp. 159-226].

Another reason to be wary of drawing substantive philosophical conclusions from Heisenberg's disturbance analysis of indeterminacy is that talk about "disturbance" or "interference" seems to beg one of the crucial questions at issue in debates over objectivity and realism in quantum mechanics. Specifically, it presupposes that the observed system has a definite value of the parameter which is the conjugate of the measured parameter (for example, a definite momentum in the case of a position measurement), for otherwise it would be unclear what, if anything, is being disturbed.

It is indeed true that, from a philosophical point of view, one of the most important implications claimed for the quantum theory is that it mandates a radical revision in our understanding of the observer-observed relationship. Bohr, Heisenberg and others have argued that the picture 
of the detached observer, which underlay the edifice of classical scientific realism, must be abandoned in favor of a model in which there is a far more intimate connection between observer and object. In fact, a case can be made for the claim that the two lose their separate identities during, and for some time following the observation interaction. This is a consequence of what is known as the nonseparability of the quantum mechanical description of interacting systems [30, pp. 75-156]. Interestingly, Wigner proposes an extremely subjectivist interpretation of quantum mechanics [31]. He adopts the account of the measurement process described above and argues that the superposition representing the instrument's state is reduced in the consciousness of the observer. Still, in all of this, and Wigner's musings about the reduction of superpositions in consciousness notwithstanding [31], it should be stressed that Bohr's position is not at all like Wigner's, and there need be no mention of observing subjects. If a conscious human observer plays any role at all, his subjective consciousness is of no consequence; for an understanding of observation in the quantum domain, we need only consider the observer as a physical system.

No doubt some confusion about this matter has been caused by the fact that Bohr occasionally speaks as if what were at issue here is the "subject-object" relationship, as when he talks of the urgent necessity, in the consideration of quantum mechanical observations, of paying "proper attention to the placing of the object-subject separation" [32, p. 79]. But a careful reading of these passages reveals that this manner of speaking is nothing more than Bohr's concession to standard philosophical parlance when he is attempting to explain to a broad audience the "epistemological implications of the lesson regarding our observational position, which the development of physical science has impressed upon us," [32, p. 78] and, in particular, when he is attempting to point up the structural analogy between the observer-observed relationship in quantum mechanics and the subject-object relationship in psychology [33]. Ordinarily Bohr speaks simply of the relationship between the observed object and the measuring instruments we employ in observation, with no mention of an observing subject. In this cleaner idiom, the need to attend to the placing of the object-subject separation becomes the

necessity of discriminating in each experimental arrangement between those parts of the physical system considered which are to be treated as measuring instruments and those which constitute the objects under investigation. [9, p. 701]

Ironically, the very passage which Popper cites from Bohr to back up his claim that the Copenhagen interpretation stresses the interference of the subject with the object under investigation, refers not to the subject, but to the measuring instruments:

\section{Indeed the finite interaction between object and measuring agencies ... entails ... the necessity of a final renunciation of the classical ideal ... and a radical revision of our attitude towards the problem of physical reality. [9, p. 697]}

The fact of the matter is that Bohr rejects, unequivocally, the suggestion that his interpretation of quantum mechanics assigns the subject any special role:

...the decisive point is that in neither case [neither relativity nor complementarity] does the appropriate widening of our conceptual framework imply any appeal to the observing subject, which would hinder unambiguous communication of experience ... in complementary description all subjectivity is avoided by proper attention to the circumstances required for the well-defined use of elementary physical concepts. [34, p. 394]

The expression "unambiguous communication" alludes to Bohr's own conception of objectivity. In his more careful moments, Heisenberg is equally clear about there being no special role for the subject in quantum mechanics:

Of course the introduction of the observer must not be misunderstood to imply that some kind of subjective features are to be brought into the description of nature. The observer has, rather, only the function of registering decisions, ... and it does not matter whether the observer is an apparatus or a human being. [35, p. 121]

Thus, though it is perhaps warranted to speak, as Popper does, of Bohr's having sanctioned an "intrusion of the observer" into quantum theory, there is no warrant for speaking, as Popper also does, of an "intrusion of the subject", as if the two kinds of intrusion came to the same thing. It should be pointed out here that Bohr was, from the start, sceptical of Heisenberg's disturbance analysis of indeterminacy [10] [32, p. 73]. As early as 1927 he was exhorting readers who wished to grasp the full significance of Heisenberg's formulae to consider not only the "possibilities of observation", but also the "possibilities of definition", meaning that we have to go beyond an analysis of the observer-observed interaction to examine the way concepts such as position and momentum are employed in the description of quantum phenomena [7, $\mathrm{p}$. 73]. 
We earlier saw that Popper's account of the etiology of subjectivism in the Copenhagen interpretation of quantum mechanics assigns a major role to the way Bohr and colleagues are supposed to have interpreted the probability calculus, the allure of the subjective interpretation of probability being cited as a principal reason for their alleged choice of a subjective epistemic interpretation of the state function. Do we have here the makings of a better argument for the charge of subjectivism? Recall that the subjective interpretation of probability amounts, roughly, to the claim that the probability function is a measure of an individual's degree of confidence in the truth of a proposition describing the event whose probability we want to know. It is subjective because different individuals could assign different probabilities to the same event. "Degree of confidence", especially when understood as something like "strength of belief", has a pronounced World 2 character. What is the evidence that Bohr and the other Copenhagen theorists subscribed to this view of probability?

Popper bases his case on the fact that many of the thinkers he associates with the Copenhagen school portray the lesson of indeterminacy as the existence of limitations on our knowledge of the properties of quantum systems. For example, Heisenberg, the source of this interpretive tradition, comments:

The uncertainty principle refers to the degree of indeterminateness in the possible present knowledge of the simultaneous values of various quantities with which the quantum theory deals. [22, p. 20]

As Popper tells it, subjectivism infected the thinking of the Copenhagen school because its members give the wrong reasons for the probabilistic character of the quantum theory, and

Foremost among these reasons is the argument that it is our (necessary) lack of knowledge - especially the limitations to our knowledge discovered by Heisenberg and formulated in his "principle of indeterminacy" or "principle of uncertainty" - which forces us to adopt a probabilistic, and consequently a statistical, theory. [1, p. 17]

As to why this leads to subjectivism, he continues:

... it is this mistaken belief that we have to explain the probabilistic character of quantum theory by our (allegedly necessary) lack of knowledge, rather than by the statistical character of our problems, which has led to the intrusion of the observer, or the subject into quantum theory. It has led to this intrusion because the view that a probabilistic theory is the result of lack of knowledge leads inescapably to the subjectivist interpretation of probability theory; that is, to the view that the probability of an event measures the degree of somebody's (incomplete) knowledge of that event, or of his "belief" in it. [1, pp. 17-18]

There can be no denying that adoption of a subjective interpretation of probability would incline one to interpret the quantum mechanical state function in a subjectively epistemic manner. But several objections can be raised to Popper's analysis of the Copenhagen attitude toward probability. To begin with, it is by no means obvious that a limitations-on-knowledge interpretation of indeterminacy leads "inescapably" to the subjective interpretation of probability. It would depend, in part, on whether the knowledge in question is of the subjective, World 2 variety or the objective, World 3 variety. If it is the latter, then, at best one might speak of an epistemic interpretation of probability, but not of a subjective interpretation, at least not in the special pejorative sense that subjective carries in Popper's vocabulary. What I am here calling an epistemic interpretation has much in common with the "logical" theory of probability, or "probability," in Carnap's terminology, which regards probability statements, such as $p(a, e)=r$, as logical truths expressing something like the degree of evidential support which a body of evidence, $e$, provides for an hypothesis, $a$. Popper considers the logical theory to be merely a "variant" of the subjective interpretation [13, §48, p. 136]. But this is a mistake, encouraged partly by some of the logical theory's proponents' having spoken of logical probability as a measure of degree of rational belief, and partly by Popper's systematic neglect of the distinction that one might, in any case, draw between belief, simpliciter, and rational belief, for talk of rational belief presumably alludes to objective norms of rationality [25] [36] pp. 43-47, 238-241]. Indeterminacy would entail limitations on subjective knowledge only if it were a consequence of an observing subject's special relationship with the observed object. But, as I have argued, Popper's attribution of this view of indeterminacy to Bohr and Heisenberg is a misreading of their positions. When indeterminacy is correctly viewed as a feature of the physical relationship between the observed object and the instruments used to measure its various properties, then whatever limitations it entails on our knowledge of quantum systems are properly seen as purely objective limitations, the same for all observers. Popper's failure to distinguish subjective and objective knowledge in the context of the interpretation 
of probability is evident in the following passage, where it is unclear which kind of knowledge is intended:

the various subjective interpretations have all one thing in common: probability theory is regarded as a means of dealing with the incompleteness of our knowledge. [37, p. 25]

On a deeper level, it is not clear why tracing the need for probabilities in quantum mechanics to our necessarily limited knowledge of quantum systems (if this is in fact what Bohr and Heisenberg do) commits one to interpreting those probabilities in an epistemic fashion. To take an example from a less controversial domain, there would be no inconsistency in my claiming that I have to describe the behavior of a tossed coin probabilistically because there are no practical means by which I could know all of the relevant initial conditions of a toss, while nevertheless insisting that what I mean when I make a probabilistic prediction about the outcome of a toss is that in a hypothetical infinitely long run of similar tosses a certain relative frequency of heads and tails would be found.

But the most serious objection to Popper's analysis is simply that, aside from Heisenberg's characterization of the uncertainty principle as referring to an "indeterminateness" in our knowledge (which, one should note, says nothing about probability, per se), there is little basis for the claim that the Copenhagen interpretation views limitations on knowledge as the explanation of the probabilistic character of quantum mechanics. In fact, Bohr explicitly rejects this account. In explaining why quantum mechanics differs essentially from statistical mechanics, where resort to a probabilistic description is generally conceded to be the result merely of a lack of complete, knowledge of all the relevant parameters of the systems under investigation (and a science in which Popper also finds significant traces of subjectivism), Bohr remarks:

...we have in each experimental arrangement suited for the study of proper quantum phenomena not merely to do with an ignoranee of the value of certain physical quantities, but with the impossibility of defining these quantities in any unambiguous way. [9, p. 699]

Bohr's position, in other words, is that we have to resort to a probabilistic description in quantum mechanics because of limitations more basic than any limits on our knowledge of the parameters of quantum systems. In another place, Bohr comments:

... the statistical character of the uncertainty relations in no way originates from any failure of measurements to discriminate within a certain latitude between classically describable states of the object, but rather expresses an essential limitation of the applicability of classical physical ideas to the analysis of quantum phenomena. [33, p. 311]

Bohr's interpretation is not a variety of the intrinsic indefiniteness interpretation. In his view, the parameters conjugate to a measured parameter are not indefinite. Instead, he would say that the concepts corresponding to these parameters do not even apply in such a context (e.g. the concept of momentum does not apply in the context of a position measurement) [38,39].

One must be cautious about imputing views on the interpretation of probability to Bohr and Heisenberg. As far as I know, neither ever made any explicit pronouncements on the interpretation question, and what little each does say about the role of probabilities in quantum mechanics can just as easily be read as suggesting an objective interpretation as a subjective one. Ultimately, the question is one of the possibilities for testing the quantum theory's probabilistic predictions, for, as Popper correctly observes, the mark of an objective interpretation of probability is its securing the objective testability of probability statements [37, p. 25]. Popper argues that the logical theory is not an objective interpretation precisely because logical probability statements are "untestable tautologies" [25, p. 357]. It is true that such statements are not subject to empirical testing, but Popper has noted elsewhere that the real test of the objectivity of scientific statements is their susceptibility to inter-subjective criticism [13, §8, p. 22] and logical probability statements fare quite well by this criterion, for we can question whether the degree of evidential support has been properly assessed, just as we can criticize a deduction in mathematics. Furthermore, Popper offers no evidence of Bohr's or Heisenberg's having said anything that suggests doubt about the testability of quantum mechanical predictions. My guess is that if we had explained the options to them, Bohr, at least, would have declared, without hesitation, that he had always understood quantum mechanical probabilities in an objective sense, as referring to the relative frequencies of results one would expect to find in repeated measurements of a given parameter on similarly prepared systems. Such an exercise being impossible, however, the best course of action is to suspend judgment. A case, of sorts, can even be made for attributing something like a propensity interpretation to Bohr; see section 5.

We must conclude that Popper has not established his claim that physics has become "a stronghold of subjectivist philosophy" under the influence of the interpretation of quantum mechanics championed by Bohr. The allega- 
tion that the Copenhagen interpretation accords a special role in quantum mechanics to the observing "subject" and the charge that it incorporates a subjective interpretation of probability are both unsubstantiated. Quotation of a few equivocal remarks by a peripheral figure like Jeans, a non-physicist like Schlick and even by a major figure like Heisenberg, who, though responsible for some of the most important formal developments in quantum mechanics, was indirectly criticized by Bohr for the superficiality of his views on interpretation, does not suffice to show that Bohr's position - the most sophisticated, subtle and systematic of the views lumped together under the Copenhagen designation - is subjectivist. This is not to say that Bohr never made mistakes, is never obscure and never inconsistent. He is guilty of all these failings. But he is not a subjectivist.

A more sympathetic reading of Bohr, and one that attends to the many differences between Bohr and the other Copenhagen thinkers, reveals that he is just as anxious as Popper to preserve the objectivity of quantum mechanics, even if his approach is somewhat different. In a discussion of the aim of science, Bohr remarks:

... our task must be to account for [human] experience in a manner independent of individual subjective judgement and therefore objective in the sense that it can be unambiguously communicated in the common human language. [40, pp. 63-64]

And of his own complementarity interpretation of quantum mechanics he says:

The notion of complementarity does not imply any renunciation of detailed analysis limiting the scope of our inquiry, but simply stresses the character of objective description, independent of subjective judgment, in any field of experience where unambiguous communication essentially involves regard to the circumstances in which evidence is obtained. [41, p. 1105]

Unambiguous communicability is not the same thing as intersubjective testability (though it might turn out that both are secured by the same conditions), but a disagreement over the definition of objectivity does not, by itself, entitle Popper to conclude that Bohr's interpretation is not objective.

Finally, what about Popper's claim that the Copenhagen interpretation asserts that "objective reality has evaporated"? He offers no independent argument for this claim, apparently on the assumption that it is implied by the subjectivism charge. Is this claim any more warranted than the latter? That Heisenberg believed that objective reality has evaporated is certain, for he is the source of the quoted remark [28, p. 100]. But this is another place where we must be careful to distinguish Heisenberg's views from Bohr's, for while Bohr is clearly critical of the presuppositions of the kind of realism associated with the world-view of classical physics, his criticism does not extend as far as Popper suggests. Bohr's position is not that there is no quantum mechanical reality, but rather that quantum mechanics forces us to reexamine and redefine the circumstances under which we can speak of the reality of the properties of quantum systems, the fundamental claim of his complementarity interpretation being that we can regard complementary properties as real only under incompatible experimental conditions, and never simultaneously [42], because

these conditions constitute an inherent element of the description of any phenomenon to which the term 'physical reality' can be properly attached [9, p. 700]

We will return to Bohr's attitude toward realism in section 4, but it should already be clear that on this issue, too, Popper's attack on Bohr is, if nothing else, a bit hasty. In his Autobiography, Popper says the following: "About Bohr I said little in Logik der Forschung because he was less explicit than Heisenberg, and because I was reluctant to saddle Bohr with views which he might not hold" [15, p. 111]. One wishes that Popper had continued to display such reserve.

There is a deep dispute between Bohr and Popper, for, as the previous quotation suggests, Bohr denies the possibility of the simultaneous definiteness of conjugate variables, while Popper asserts that they are always simultaneously definite, and this assertion is a central part of Popper's own interpretation of quantum mechanics. Moreover, to someone who believes in simultaneous definiteness, the claim that we cannot simultaneously know both the position and momentum of a system with arbitrary precision might appear to say more about our knowledge than about the world, and hence, might appear as a kind of subjectivism. But not only would such an inference run the risk of the confusion, discussed above, between the objective and subjective senses of knowledge, it would also be objectionable on the ground that, since it is based on the assumption of simultaneous definiteness, it thus ignores the possibility that the apparent limitations on our knowledge are a genuine reflection of some important features of objective, quantum mechanical reality. 


\section{Simultaneous definiteness and the statistical scatter interpretation of indeterminacy}

As we saw above, Popper believes that physicists were led down the garden path of subjectivism by their desire to reconcile the theory's probabilistic character with their belief that it is also a fundamental theory, a theory of individual quantum systems. At the time he wrote Logik der Forschung, Popper believed that the way to avoid subjectivism is to give up the latter belief. Accordingly, he proposed an objective statistical interpretation of quantum mechanics, which holds that the theory is true only of ensembles or aggregates of systems [13, p. 234], and the centerpiece of this alternative is the proposal that we view the Heisenberg indeterminacy formulae as neither the consequence of limitations on measurement nor an expression of intrinsic indefiniteness, but as statistical scatter relations [13, §75]. Popper later believed that we have an objective probabilistic theory of individual systems, which is embodied in his later propensity interpretation of quantum mechanics, and thus he gave up the claim that quantum mechanics only refers to ensembles; but he still defended the statistical scatter view of indeterminacy and all that it presupposes [1, pp. 20-21].

To interpret the indeterminacy formulae as statistical scatter relations is, first, to assume that atomic systems can possess simultaneously sharp values of their conjugate parameters, and, then, to construe the formulae as expressing lower limits on the scatter of the results of measurements of these parameters on any ensemble of such systems. For example, imagine that we have a device, such as a screen with a slit in it, which selects from a beam of incoming particles only those whose (definite) positions are confined to the region $q_{x} \pm \frac{1}{2} \Delta q_{x}$. This selection defines an ensemble of particles according to their positions. The scatter interpretation would then imply that the (definite) results of measurements of the momenta of the particles of this ensemble must be scattered over a range no smaller than $\Delta p_{x} \geq \frac{\hbar}{2 \Delta q_{x}}$. In Popper's words, the Heisenberg formulae, construed thus, "mean only that there are limits to the statistical homogeneity" of the results [1, p. 21].

What is really controversial is not the scatter interpretation per se, but the assumption upon which it rests, that, contrary to the standard interpretations of indeterminacy, simultaneously precise conjugate parameters are possible. In a bold gesture, Popper claims not only that this assumption is compatible with quantum mechanics, but also that simultaneously precise measurements of these parameters are possible and, in fact, necessary for testing the statistical predictions of the theory [1, p. 20]. All of these assertions are questionable.

Popper's argument regarding simultaneously precise measurements starts with a critique of some early thought experiments which Heisenberg used to illustrate his own interpretation of indeterminacy. The experiments use standard methods of measuring either the position or momentum of an atomic system, and they all have this much in common: when the physical details of the measurement procedure are examined, say in the case of a position measurement, it is allegedly found that the measurement necessarily produces a mechanical disturbance of the observed system's momentum of precisely the magnitude required to satisfy the indeterminacy relations [22, pp. 21-30].

A typical way to measure momentum (the product of mass and velocity) is to combine two position measurements. Knowing a particle's mass, we measure its position at two different times and divide the resulting distance by the time of flight to get the velocity, which we multiply by the mass in order to get the momentum. If we allow enough time to elapse between the position measurements, momentum measurements of theoretically unlimited accuracy are possible. However, as Heisenberg points out, the accuracy thus obtained pertains only to the particle's momentum before the second measurement, because the momentum uncertainty necessarily engendered by this measurement renders the result computed from the two measurements useless for predictions of the particle's momentum after the second position measurement. Heisenberg concludes that while retrodictive momentum measurements of unlimited accuracy are possible, predictive measurements must always conform to the restrictions of the indeterminacy formulae [22, p. 25]. (The terminology - predictive and retrodictive measurements - is Popper's, not Heisenberg's [1, p. 25]).

Popper's criticism of Heisenberg first took the form of a head-on attempt to show, by means of another thought experiment, that predictive measurements violating the indeterminacy relations were also possible [43] [13, §77]. But shortly after the publication of the experiment Einstein detected an error in it [13, §*xii]. With the failure of this approach, Popper shifted the focus of his criticism to Heisenberg's concession that retrospective violations of the indeterminacy principle are possible. Heisenberg explained his attitude toward such violations as follows:

This formulation makes it clear that the uncertainty relation does not refer to the past; if the velocity of the electron is at first known and the position then exactly measured, the position for times previous to the measurement may be calculated. Then for these past times $\Delta p \Delta q$ is smaller than the usual limiting value, but this 
knowledge of the past is of a purely speculative character, since it can never (because of the unknown change in momentum caused by the position measurement) be used as an initial condition in any calculation of the future progress of the electron and thus cannot be subjected to experimental verification. It is a matter of personal belief whether such a calculation concerning the past history of the electron can be ascribed any physical reality or not. [22, p. 20]

Popper welcomes the concession, but quite rightly attacks Heisenberg's crudely positivistic interpretation of the significance of retrodictions. Referring to the last sentence of the previous quotation. Popper remarks:

But it is not a matter of personal belief: the measurements in question are needed for testing the statistical laws (1) and (2) [the indeterminacy formulae] that is, the scatter relations ... To question whether the so ascertained "past history of the electron can be ascribed any physical reality or not" is to question the significance of an indispensible standard method of measurement (retrodictive, of course); indispensible, especially, for quantum mechanics. But once we ascribe physical reality to measurements for which, as Heisenberg admits, $\Delta p \Delta q \ll h$, the whole situation changes completely: for now there can be no question whether, according to the quantum theory, an electron can have a precise position and momentum. It can. [1, p. 27]

Up to a point, Popper is right. If simultaneously precise retrodictive measurements are possible, we have little choice but to ascribe reality to their results. But he should not have been so quick to trust Heisenberg's authority regarding the existence of such measurements. Popper's argument for simultaneous definiteness rests largely on Heisenberg's analysis. But maybe Heisenberg was wrong.

In fact, I think Heisenberg and, thus, Popper are both wrong, and the error in their reasoning is simple to detect. Consider the case of a retrodictive momentum measurement, one consisting of two successive position measurements. The putative violation of indeterminacy arises because we can presumably infer the particle's momentum at any time prior to the second position measurement, and thus, in particular, at the time of the first position measurement, with arbitrary precision. Whatever the uncertainty attaching to the first position measurement, $\Delta q_{x}$, it would follow that we could infer a momentum at the time of that measurement more precise than permitted by the indeterminacy relations, that is, with $\Delta p_{x} \ll \frac{\hbar}{2 \Delta q_{x}}$ (the reasoning, and the criticism, are the same if we look at the particle's position and momentum immediately prior to the second position measurement). But this conclusion is incorrect. What we infer from the two position measurements is not the instantaneous momentum of the particle, but only its average momentum during the interval. If we assume that the momentum was constant during that interval, then the average and instantaneous momenta would be equal, but otherwise, no conclusion about the momentum at any specific time is licensed. Of course, one can typically advance theoretical arguments for assuming the approximate constancy of the momentum, such as the absence of any external forces. But notice that the assertion, for whatever reason, of a precisely constant momentum - the necessary hidden assumption in the argument for retrospective violations of indeterminacy - again begs the very question at issue, in as much as it would be the assertion of constant definite momentum, which is exactly what the indeterminacy relations are believed by Bohr and others to disallow (unless, of course, the position is completely indeterminate).

Popper, on his own, adduced other examples of putative simultaneously precise measurements of conjugate variables. I think they all fail, but for now, I will only examine the most interesting one. It draws upon a thought experiment first proposed by Einstein, Podolsky and Rosen (EPR) in 1935 as part of their famous attempt to prove the incompleteness of quantum mechanics [44], but I will discuss Popper's analysis as it applies to another experiment, suggested by David Bohm, which is simpler than the EPR experiment, though formally identical to it in all relevant respects [45, pp. 614-622]. The simplicity of the Bohm version of the experiment is largely due to its employing discrete variables, rather than the continuous position and momentum variables of the EPR experiment. Consider the following situation. A spin-zero particle decays into two spin- $\frac{1}{2}$ particles, $A$ and $B$. Assume that we have filters which allow us to select pairs of decay products traveling in opposite directions without affecting their spins, and that we have devices (called Stern-Gerlach apparatuses) which allow us eventually to measure the spins of the decay particles along any spin axis orthogonal to the particles' paths. The quantum mechanical description of the decay process entails that total spin is conserved. Thus, the spin of the original particle being zero, the sum of the spins of the decay products must also be zero along all spin axes. Since the decay products are spin- $\frac{1}{2}$ particles, the spin conservation principle implies that if we measure the spin of particle $A$ along the $z$-axis and get a result of $-\frac{1}{2}$ we can infer that the $z$-spin of particle $B$ is $+\frac{1}{2}$. Popper's argument would be that it is possible (at least by accident) to measure simultaneously the $z$-spin of $A$ and the $y$-spin of $B$, and then to infer values for the $y$-spin 
of $A$ and the $z$-spin of $B$ by conservation of spin. Thus, he would conclude, we can make simultaneously precise measurements of both the $z$-spin and $y$-spin of $A$ and $B$, even though spins along orthogonal axes, being conjugate variables, are supposedly not simultaneously measurable. And if we can measure both components of spin, we have little reason to deny their reality. For Poppers actual argument, which assumes the original EPR version of the thought experiment, see [13, §*xi].

Simultaneous measurements of the $z$-spin of $A$ and the $y$-spin of $B$ are possible. What is questionable about Popper's analysis is, again, the inferences drawn from the results of these measurements. The particular problem in the present case is the uncritical use of conservation arguments. Spin conservation is implied by the quantum formalism only in the following restricted sense: if the same component of spin is measured on both decay products, then the probability of the results summing to zero is equal to unity. By itself, the formalism licenses no inferences about spin conservation in the case of measurements of different components, which is the case Popper contemplates. In order to invoke conservation arguments in this case, additional assumptions are needed. Specifically, one must assume precisely what the possibility of simultaneous measurements are held to establish, namely, that each particle actually possesses a definite spin along every axis, and not just along the axis for which the spin is measured. (One must also assume that these definite spins are correlated as required by the conservation principle.) Only thus can a direct measurement of, say, the $z$-spin of $A$ be held also to constitute a measurement of the $z$-spin of $B$.

What the foregoing reflections show is that Popper cannot argue for the simultaneous definiteness thesis by appealing to the possibility of simultaneous measurements of conjugate parameters, because his putative examples of the latter are interpretable as simultaneous measurements of the necessary sort only if one assumes simultaneous definiteness. In other words, Popper is arguing in a circle. There would be nothing particularly vicious about this circle if there were independent evidence for the definiteness thesis, but such evidence has not been provided, and none is likely to be forthcoming. Indeed, in this connection Popper's exploitation of the EPR-type thought experiment is especially ironic, because that experiment can be turned into an empirical argument against both the simultaneous definiteness thesis and a broad class of realistic interpretations of quantum mechanics.

Einstein, Podolsky and Rosen sought to use their thought experiment to prove that quantum mechanics is incomplete. Their argument begins with a crucial assumption, namely, a criterion of physical reality:
If, without in any way disturbing a system, we can predict with certainty (i.e., with probability equal to unity) the value of a physical quantity, then there exists an element of physical reality corresponding to this physical quantity. [44, $\mathrm{p}$. 777]

Then they reason, in effect, that the possibility of indirect, non-disturbing measurements of the kind sketched above, such as a measurement of the $z$-spin of $B$ carried out by first measuring the $z$-spin of $A$ and then inferring a value for $B$ by the conservation principle, shows that, without disturbing a system, we can predict with certainty the values of two of its conjugate variables. This is because we could have measured either the $z$-spin or the $y$-spin of $A$, and thus could have made a prediction of the value of either component of $B$ 's spin, and whichever component we predict, a subsequent direct measurement of it would confirm our prediction. But then it follows, according to the reality criterion, that both the $z$-spin and $y$-spin of $B$ are real, and since the orthodox quantum theory rules out the simultaneous definiteness of conjugate variables, one would have to conclude that quantum mechanics is incomplete. In reply, Bohr pointed out that the two indirect measurements cannot be executed simultaneously, since the necessary experimental arrangements are incompatible (for example, the Stern-Gerlach apparatus which measures $A$ 's spin cannot be oriented in such a way as to measure simultaneously spin along two different axes), and since he held that the experimental conditions

constitute an inherent element of the description of any phenomenon to which the term 'physical reality' can be properly attached. [9, p. 700]

he therefore denied that the EPR argument established the simultaneous reality of conjugate variables.

Because Bohr makes his own strong assumptions about the circumstances under which we can speak of the reality of quantum properties, his reply is hardly a refutation of the EPR argument. But something close to a refutation is possible, or, at least, a very uncomfortable dilemma can be forced upon EPR. Because of the thought experiment's basic symmetry, EPR's premises actually imply the simultaneous reality of the conjugate parameters of both $A$ and $B$ (which is precisely what Popper wants to assert), and this implies, in turn, that each of the systems has its own separate physical state. That EPR are committed to this assumption was first suggested in [46]; but for a detailed defense of the attribution one should consult my dissertation [47]. A helpful discussion of these issues may be found in [30, §8-9]. If there is any doubt that Popper agrees with this consequence of the EPR point of view, consider the following remark: 
Thus the so-called "paradox" of Einstein, Podolsky, and Rosen is not a paradox but a valid argument, for it established just this: that we must ascribe to particles a precise position and momentum, which was denied by Bohr and his school [1, p. 28]

If one believes, in this sense, that the two systems have their own "independent reality" from the time they cease to interact, it would seem to follow that whenever we perform a direct measurement on one of the systems, the result will depend solely on the properties of that system. But while this might appear to be an obvious fact about measurement, it can be shown that the locality assumption (i.e., the results of a measurement depend solely on the local properties of the measured object) necessarily leads to predictions for a class of correlation measurements, which can be performed with the EPR-type experimental set-up, that differ markedly from the predictions of the quantum theory. Moreover, experiments of this type have been performed, and the results confirm, to a high degree, the quantum mechanical predictions. The basic theorems, which are the work of J. S. Bell, and the experimental results are nicely summarized in [48]. (For an elegant, non-technical treatment, one might look at [49] by David Mermin; his speculations about the philosophical implications of Bell's work should, however, be taken with a grain of salt). What this shows is that one must either give up the realistic thesis that previously interacting systems go into separate physical states (which, of course, entails a denial that they go into separate, definite states), or assume the existence of nonlocal effects, that is, superluminal signals by means of which the result of a measurement performed on one of the systems can be influenced by our choice of which parameter we will measure on the other system. The hypothetical signals would have to be faster than the speed of light because we can design the experiment in such a way as to insure that there would not be adequate time for a subluminous signal to travel from one Stern-Gerlach apparatus to the other. Nor will it do to argue, if one finds the latter option unsavory, that while the independent reality of the two systems might not be found at the level of the traditional quantum mechanical properties, such as spin, it may exist at a deeper level, say at the level of some hypothetical "hidden parameters". For the negative result is quite general: any technique, which makes measurement results solely dependent on a system's local properties, be they hidden or not, will yield the wrong predictions [50]. Quantum mechanics avoids nonlocal effects precisely by refusing to assign separate (let alone definite) states to the two systems, describing them instead by means of a single state function for the composite system, $A+B$, a state function which cannot be decomposed as a product or sum of separate state functions for $A$ and $B$.

These results must be discomfiting to Popper. Since the simultaneous definiteness thesis implies that previously interacting systems have separate physical states, the only way Popper can continue to maintain that thesis, and to assert that it is compatible with quantum mechanics, is to assume the existence of nonlocal effects. While no one has yet provided any independent evidence for such, effects, one may, of course, still assert their existence as a conjecture, but it should be noted that the price for securing compatibility with quantum mechanics is an assumption that is incompatible with the relativity theory's assertion that the speed of light is the upper limit for the propagation of physical effects. And, to repeat, this problem is quite general: any attempt to give a realistic interpretation of quantum mechanics will be saddled with nonlocality as long as it attributes an independent reality, at whatever level, to previously interacting systems. The philosophical implications of the work on nonlocality are discussed, with slightly different emphases, in [51,52]. The latter essay should be read with some caution. d'Espagnat argues that the predictions which have been refuted by experiment are actually entailed by three premises: realism, locality and the "free use of induction". Supporters of Popper might take solace from this claim, arguing that realism can be saved by denying the third premise, something Popper would have us do anyway. But I think d'Espagnat is simply mistaken in asserting that induction is a necessary premise in the derivation; the crucial extrapolation which he says [52, p. 177] has to be supported by induction could just as well be construed as a highly corroborated conjecture, and not as an inductive extrapolation from limited evidence. Caution is also advised regarding Shimony's rather instrumentalistic reading of Bohr's position.

I have argued that Popper's arguments for the possibility of simultaneously precise measurements of conjugate variables are flawed, and that the thesis that quantum systems possess simultaneously definite values of conjugate variables (whether measurable or not) can be maintained only at a considerable price. What, finally, of his claim that measurements more precise than those presumably allowed by the indeterminacy relations are needed to test those relations? Do the former negative results mean that the indeterminacy relations cannot be put to a test? I think not. If the Heisenberg formulae were scatter relations of the sort Popper claims, then simultaneously precise measurements would be required to test them. But the difficulties with the simultaneous definiteness thesis argue against this interpretation. The indeterminacy formulae are better viewed simply as relating the expected scatter or standard deviation in the results of 
measurements of conjugate observables on similarly prepared systems. Nothing requires that the measurements be carried out on the same system. Thus, one can measure the position of half the members of an ensemble of similarly prepared systems, and then the momentum of the other half. If, according to ordinary rules of statistical inference, the product of the standard deviations of the two sets of measurements is significantly lower than the minimum posited by the indeterminacy relations, then those relations can be considered falsified; if the product is not significantly lower, then the relations have passed this particular test. No simultaneous measurements are possible, none are required.

\section{The propensity interpretation of quantum mechanics}

The latest stage in the development of Popper's thinking on the quantum theory is represented by his propensity interpretation of probability and quantum mechanics. In one sense this idea betokens a radical shift by Popper, for while he formerly thought that physicists were pursuing a will-o'-the-wisp in attempting to forge an objective probabilistic theory of individual events, he later believed that the propensity theory accomplishes precisely this goal. But in a more important sense, no shift at all is indicated, for Popper's purpose in introducing the concept of propensity is the same as it was when he advocated a statistical interpretation of quantum mechanics: the defense of objectivity and realism. The propensity interpretation's credentials as a candidate for a realistic interpretation of quantum mechanics are impressive, and its further refinement is an important item on the agenda for research into foundational questions. But there are some confusions in Popper's account of the propensity interpretation, and some of his claims on its behalf are extravagant. Moreover, I will argue that when the negative results of section 4 are taken into account, a genuine rapprochement between the propensity interpretation and Bohr's complementarity interpretation is no longer out of the question.

Popper says that he developed the propensity interpretation from a criticism of the way the relative frequency interpretation handles the probability of individual or singular events. (Remember that it was this difficulty which, according to Popper, led to the Copenhagen interpretation's allegedly subjective view of quantum mechanical probabilities. Popper gives an account of the development of the propensity interpretation in [1, pp. 30-34] [37] [53]. The latter two treatments emphasize the development from the frequency theory). Popper asks us to consider a very long actual sequence of tosses of a loaded die, for which the probability of turning up a six is $\frac{1}{4}$, and to imagine interspersed among these tosses, at unknown places, a few tosses of a fair die. The relative frequency of sixes in this sequence will still be virtually indistinguishable from $\frac{1}{4}$, the more so the longer we imagine the sequence to be, and thus there is no obstacle to our continuing to assert that the probability of throwing a six on a toss of the loaded die is $\frac{1}{4}$. What about the probability of getting a six on one of the throws of the fair die? We still want to assert that this probability is $\frac{1}{6}$, even though the toss is a member of an actual sequence in which the relative frequency of sixes is $\frac{1}{4}$. But why? According to Popper, the frequency theorist can only answer that it is because the toss of the fair die, though a member of the aforementioned actual sequence, is also a member of a virtual sequence of identical tosses, that is, a virtual sequence of tosses of a fair die, and the probability of getting a six on a toss of the fair die is properly assessed by considering the latter sequence. This may appear, at first glance, to be just a slight modification of the frequency theory, specifically, a stipulation that the only admissable sequences for defining probabilities are sequences of repeated similar trials, or, in other words, sequences characterized by repeated realizations of a set of generating conditions. But Popper argues that this really represents "a transition from the frequency interpretation to the propensity interpretation," because "probability may now be said to be a property of the generating conditions" [37, p. 34]. His reasoning is as follows:

$\ldots$ if the probability is a property of the generating conditions - of the experimental arrangement - and if it is therefore considered as depending upon these conditions, then the answer given by the frequency theorist implies that the virtual frequency must also depend on these conditions. But this means that we have to visualise the conditions as endowed with a tendency, or disposition, or propensity, to produce sequences whose frequencies are equal to the probabilities; which is precisely what the propensity interpretation asserts. [37, p. 35]

A clearer way to put Popper's point would be to note that the frequency interpretation gives us no reason for choosing one virtual sequence over another, say the virtual sequence of tosses of the fair die rather than a virtual sequence of tosses of various dice, some of them loaded. The propensity interpretation gives us a reason by making probability a property of the experimental conditions. In Popper's eyes, the chief virtue of the propensity interpretation, and the characteristic that makes it useful in giving an objective, realistic interpretation of quantum mechanics, is that it enables us to regard probability 
as a real physical property of the single physical experiment or, more precisely, of the experimental conditions laid down by the rule that defines the conditions for the (virtual) repetition of the experiment. [1, p. 33]

Various critics have taken Popper to task for obscurities in his formulation of the propensity interpretation, one of the main worries being that it is still not clear how the propensity theory differs from a frequency theory [54,55]. The likely cause of the critics' worry is Popper's ill-considered claim that the propensity interpretation regards probability statements "as statements about frequencies in virtual (infinite) sequences of well characterized experiments," which he thinks is the same as regarding them "as statements about some measure of a property ... of the whole experimental arrangement" [1, p. 32]. Indeed, if we take relative frequencies in virtual sequences as measures of propensity, then the distinction between the two interpretations blurs. But Popper's claim is simply a mistake, for while the propensity theory may have been developed through reflection on the frequentist's talk of virtual sequences, nothing requires that we take relative frequences in virtual sequences as the measure of propensities.

One can formulate the propensity interpretation as the thesis that probabilities are theoretical primitives, which are implicitly defined by a suitable set of axioms for the probability calculus, and which we associate with experimental arrangements or generating conditions, either on the basis of experience or as a result of considerations of physical theory, in order to characterize the tendency or propensity of an arrangement to produce outcomes of a certain sort. Probability statements, thus construed, would be tested, as Popper notes, by actual statistical frequencies [1, p. 32], but to say this is not to say that the statements are interpreted in terms of frequencies. Interpreting a formal system like the probability calculus is a matter of finding models for it. Probabilities might be modeled by relative frequencies; they can just as well be modeled by propensities.

To be sure, Popper has not given a general, formal definition of propensity, comparable to the ones that can be given for relative frequency, but that is not necessary in order to claim that propensities provide a model for the probability calculus. All that is required is an unambiguous rule for assigning numerical values (Popper calls them weights) [1, p. 32] to the propensities, a rule which thus allows us to define a function that can be shown to satisfy the probability axioms. Nor is it right to demand one rule and one definition for all domains of inquiry. Why, for example, should we expect biological propensities and physical propensities to be measured in the same way? How they are to be measured is a task for biological and physical theory to decide.

In the case of quantum mechanics, the rule is provided by Born's statistical interpretation of the state function. More specifically, the quantum mechanical rule may be formulated thus: let $\mathcal{H}$ be the Hilbert space associated with a system, $S$, whose state is represented by the vector $|\psi\rangle$ in $\mathcal{H}$; let $\hat{A}$ be a Hermitian operator on $\mathcal{H}$ corresponding to the observable of interest; and let $\hat{P}_{A}(x)$ be the projection operator onto the subspace of $\mathcal{H}$ spanned by the eigenvectors of $\hat{A}$ corresponding to the eigenvalue $x$. The probability that a measurement of the observable represented by $\hat{A}$ will yield the value $x$ is given by: $\left\langle\psi\left|\hat{P}_{A}(x)\right| \psi\right\rangle$. Only if we have no theory to draw upon need we resort to frequencies for an estimate of the numerical value of a propensity. But here it is misleading to speak of frequencies as providing a measure of the propensity; we can assume that the measure will be provided by some as yet undiscovered theory (or one too complicated to bother with, for practical purposes, as in the case of dice rolling) and that observed, actual frequencies suggest what that measure would be. In no case need we resort to virtual frequencies.

One of the most sensible features of the propensity interpretation is the insistence that propensities be viewed not as properties of individual objects, such as a die by itself, but as relational properties of whole experimental arrangements. As Popper notes, the probability of throwing a six with a loaded die does not depend solely on the die; the weaker the gravitational field, for instance, the more this probability will approach that for a fair die [53, p. 68]. Nor does the probability of a coin's landing heads-up depend solely on the coin; if we toss it on a flat surface, the probability is $\frac{1}{2}$ but if we toss it on a surface containing a number of slots which might catch the coin on edge, the probability of heads will be less than $\frac{1}{2}$ [1, p. 39].

But Popper goes too far when he suggests that relativizing propensities to experimental contexts at last provides a solution to the wave packet reduction problem in quantum mechanics. His argument is that if we view quantum mechanical probabilities as propensitifes, wave packet reduction is seen to be identical with a trivial, non-puzzling, and thoroughly classical phenomenon that can be demonstrated with a pin board [1, pp. 33-36]. Assume that we start with a symmetrical pin board. The more balls we role down, the closer their resulting distribution will approximate a normal distribution, which can be taken to represent the probability distribution, $p\left(a, e_{1}\right)$, for a single ball's reaching a specific place on a specific trial. If we vary the experimental situation by looking only at those balls which happen to hit a specific pin (which Popper says is like performing a position measurement on the balls), we get a different final distribution, which 
may represent the probability distribution, $p\left(a, e_{2}\right)$, for these balls' reaching a specific place. Popper contends that exactly the same kind of thing happens in the case of quantum mechanical measurement, and thus that the wave packet collapse is to be explained as nothing more than the result of a change in the experimental conditions: before a measurement is performed, the theory predicts one probability distribution, after the measurement it predicts another distribution; the difference is that the performance of a measurement changes the experimental situation.

Two comments are in order. First, as Popper's critics have noted [54, pp. 466-467], and as Popper himself, in effect, conceded, the key to this approach to wave packet reduction is not the propensity interpretation, but the recognition that the relevant probability distributions represent relative or conditional probabilities, rather than absolute ones, and this point could just as easily have been made by a frequency theorist [14, p. 73]. The move to conditional probabilities certainly appears more natural from the propensity point of view, but nothing precludes the frequentist's offering the same solution.

Second, and more importantly, Popper's suggestion does not go far enough. Even von Neumann's solution of the reduction problem by means of the projection postulate recognizes that different probability distributions are called for before and after a measurement. What makes the projection postulate objectionable is that it posits a principle for the evolution of states during observation which differs dramatically from the principle of evolution under other circumstances. As it stands, Popper's analysis of wave packet reduction is open to the same objection. To be sure, we are given a reason for picking a new probability distribution after the measurement is performed, But if the propensities which these probability distributions are supposed to represent are real properties (even of the whole experimental arrangement), we ought to be able to trace their continuous temporal evolution, and this Popper has not told us how to do. My guess is that a wholly satisfactory solution will be achieved only when the propensity interpretation's hint that quantum mechanical reality resides in the whole experimental arrangement is taken up in a thoroughgoing reformulation of the quantum formalism, a reformulation that makes the state function itself a property of the whole experimental arrangement. The standard formalism treats the state function as a context-independent property of the system. Popper, himself, seems to be a bit confused on this point. In one place he says "the $\psi$ function describes physical realities", portraying this as the lesson of the propensity interpretation [53, p. 69]. But that interpretation regards propensities, which are properties of whole experimental arrangements, as real. The $\psi$ function, as standardly defined, is not a property of whole experimental arrange- ments, and thus the propensity interpretation does not entail that the $\psi$ function describes reality. One could take an instrumentalistic attitude toward the $\psi$ function and still regard quantum mechanical propensities as real.

Popper quotes Heisenberg saying that the reduction represents a transition from the possible to the actual [1, p. 37], a transition which is completed when "the actual is selected from the possible, which, is done by the 'observer"' [12, p. 23]. What encourages Popper in thinking that the propensity interpretation already provides a complete solution to the wave packet reduction problem is his belief that the problem is solely a result of the Copenhagen interpretation's alleged grant of special status to the observer in quantum mechanics (the idea being that it is the observer's intervention during measurement that brings about the reduction). Showing that it can be explained, instead, merely as the result of a change in experimental conditions surely does away with any need to invoke the observer. But, as I argued in section 3 , this is a misreading of the Copenhagen interpretation, or, at least, of Bohr's philosophy. And, in any case, wave packet reduction involves deeper formal issues. Popper's claim that the propensity idea solves the wave packet reduction problem is but part of his larger claim that it helps us to view quantum mechanics as a statistical theory, and thus

takes the mystery out of quantum theory ... by pointing out that all the apparent mysteries would also involve thrown dice, or tossed pennies - exactly as they do electrons [53, p. 68]

Patrick Suppes has criticized the assertion that quantum mechanics is a statistical theory on the grounds that, in it

the joint distribution of noncommuting random variables [such as position and momentum] turns out not to be a proper joint distribution in the classical sense of probability [55, p. 771]

Suppes is correct, but it is important to note that his conclusion depends on our calculating the quantum mechanical joint distribution from the standard, contextindependent, quantum mechanical state function. I suspect that if we could reformulate quantum mechanics, as per my suggestion, in such a way as to make the state function a property of whole experimental contexts, we would then get classical joint distributions. Thus, I think Popper is wrong in believing that a simple reinterpretation of quantum mechanics reveals it to be a classical, statistical theory. But the propensity interpretation points the way to a reformulation of the theory that might fit this description.

Another claim that Popper makes for the propensity interpretation is that it solves the problem of the wave- 
particle relationship, and thus presumably, leads us out of "the great quantum muddle". This claim, too, needs to be scrutinized. In general terms, the muddle consists in mistaking a property of a probability distribution function for a property of either the outcomes (such as a toss of the die yielding a six) whose probability that function provides, or of some element of the system (such as the die, itself) to which those outcomes occur. Physicists make this kind of mistake whenever they say things like "an electron is both a particle and a wave", for what is wave-like is not the electron itself, but the electron's state function, $\psi(x)$, and the resulting probability distribution for its position, given by: $p(x)=\psi^{*}(x) \psi(x)=|\psi(x)|^{2}$. Others have criticized the claim that electrons are waves in any real physical sense, pointing out, for example, that we are dealing here not with waves in 3-dimensional physical space, but waves in an abstract, multi-dimensional parameter space, as evidenced by the fact that the probability distribution for the electron's momentum is also, usually, wave-like. But Popper has identified the basic confusion more clearly than perhaps any other writer [1, pp. 18-20].

However, as in the case with wave-packet reduction, the propensity interpretation does not seem to be essential to the clarification. A frequency theorist could also warn us not to confuse the shape of the distribution of balls on a pin board with the shape of the balls themselves. A propensity theorist might be less likely to fall into the confusion in the first place, but clear thinking is the real safeguard, not adherence to a particular interpretation of probability.

Popper's own view of the relationship between particles and waves avoids the "quantum muddle", but is flawed in another way. He says that "particles are important objects of experimentation" whereas the often waveshaped probability distributions are, on the propensity interpretation, "properties of the experimental arrangement" [1. p. 39]. What we have, then, is not a relationship between particles and waves, but one between "particles and their statistics" [1, p. 38], and it is misleading to speak of a duality between them. Popper is right that talk of duality is misleading. But so is talk of the "relationship between particles and their statistics" and this for two reasons. First, as Popper himself has stressed, propensities are properties of whole experimental arrangements, not of the particles investigated by those arrangements, so the crucial relationship is one between experimental arrangements and their statistics, not particles and their statistics, for, properly speaking, particles themselves have no statistics. Second, Popper's attitude toward particles is wholly classical, except, of course, for his agreeing that they do not obey deterministic laws. In particular, as was pointed out in section 4, he views particles as real entities endowed at all times with a complete set of simultaneously definite properties. But, this view of particles is untenable, or, at least, if the statistical predictions of quantum mechanics are correct (and we have no reason to doubt them), Popper's view of particles can only be maintained at the expense of assuming nonlocal effects, which means contradicting the theory of relativity. In sum, particles, as Popper understands them, might not exist at all.

Popper asserts that both particles and propensities are real [1, p. 39]. In light of the difficulties plaguing the simultaneous definiteness thesis, I would urge Popper to give up the claim about the reality of particles, and rest his realistic interpretation of quantum mechanics solely on real propensities. The propensity interpretation does not require us to talk of particles. All we need speak of are experimental arrangements and their propensities to yield certain kinds of outcomes when certain kinds of measurements are conducted with them. This does not entail denying the reality of atomic objects, it simply means that we would consider them real only as constituents of experimental arrangements.

Such a reworking of Popper's propensity interpretation opens up, finally, the possibility of a rapprochement of sorts between Popper and Bohr. Nowhere does one find Bohr speaking of "propensities". The interpretation of the probability calculus is the kind of formal issue in which he displays little interest. And, of course, Bohr's opaque prose style makes it difficult to say exactly what his views are on a number of issues. But in his own way he too emphasizes that solving the problem of objectivity in quantum mechanics requires our according whole experimental arrangements a central role in the interpretation of the theory. He also sees that the statistical character of the theory is a consequence of the fact different outcomes are ordinarily obtained upon repetition of one and the same experiment.

Recall that, for Bohr, objectivity is a matter of the unambiguous communicability of experience. What makes quantum mechanics unique is that here an unambiguous account of experience is made more difficult to achieve because of the intimate relationship between measuring instruments and objects of investigation, which means that we have to pay special attention to experimental arrangements. Bohr says:

While, within the scope of classical physics, the interaction between object and apparatus can be neglected or, if necessary, compensated for, in quantum physics this interaction thus forms an inseparable part of the phenomenon. Accordingly, the unambiguous account of proper quantum phenomena must, in principle, include a description of all relevant features of the experimental arrangement. [34, p. 391] 
More specifically, Bohr argues that we should interpret quantum mechanics as referring only to what obtains within well-defined experimental arrangements:

It is certainly far more in accordance with the structure and interpretation of the quantum mechanical symbolism, as well as with elementary epistemological principles, to reserve the word "phenomenon" for the comprehension of the effects observed under given experimental conditions. [33, p. 316]

What makes quantum mechanics an irreducibly probabilistic theory is the fact that even after we have specified as completely as possible what constitutes a repetition of the same experiment, we typically find that the same experiment yields different results. The next-to-last quotation continues:

The very fact that repetition of the same experiment, defined on the lines described, in general yields different recordings pertaining to the object, immediately implies that a comprehensive account of experience in this field must be expressed by statistical laws.

And in the same essay, Bohr remarks about the quantum formalism that

its physical interpretation finds expressions in laws, of an essentially statistical type, pertaining to observations obtained under given experimental conditions. [34, p. 390]

It is but a short step to the conclusion that the reality which the quantum theory describes is precisely the tendencies or propensities of experimental arrangements to produce results of a certain sort.

In his intellectual autobiography, Popper makes the following claim about the reception of his propensity interpretation:

I remember that the theory was not well received to start with, which neither surprised nor depressed me. Things have changed very much since then, and some of the same critics (and defenders of Bohr) who at first dismissed my theory contemptuously as incompatible with quantum mechanics now say that it is all old hat, and in fact identical with Bohr's view. [15, p. 180]

I want to make it clear that I am not claiming that Popper's interpretation of quantum mechanics is identical with Bohr's. There are interesting similarities, which become especially evident when one discounts Popper's misreading of Bohr's philosophy as a variety of subjectivism. But equally significant are the differences, which center around Bohr's idea of complementarity, and derive from a fundamental disagreement over the relationship between different experimental arrangements. Popper's simultaneous measurability thesis implies the compatibility of all experimental arrangements. Bohr, on the other hand, argues that the experimental arrangements necessary for the measurement of pairs of conjugate parameters are mutually exclusive, even though both parameters are, in a sense, equally necessary for giving a complete account of atomic objects. To be accurate, Popper effectively concedes the exclusiveness of the experimental arrangements for a certain kind of measurement of conjugate parameters, namely, the kind he designates a "preparation of state". The passage of a beam of particles through a narrow slit would be such a measurement, in as much as it tells us something about the positions of the particles. This kind of measurement corresponds to what was earlier called a "predictive measurement" [1, p. 21]. The mutual exclusiveness of experimental arrangements is what lies at the root of complementarity, and thus it is also the deep reason for Bohr's denial of the simultaneous reality of conjugate parameters. In Bohr's opinion, it is the novel, complementary relationship of quantum mechanical phenomena that makes quantum mechanics unique among physical theories, including other statistical theories [9,34]. If the propensity interpretation is detached from the simultaneous definiteness thesis, it can become the natural ally of the complementarity interpretation, and one can hope that such an alliance would lead to deeper insight into the implications of quantum mechanics.

Does Popper's propensity interpretation accomplish the original aims of his program in the philosophy of quantum mechanics? In one sense, yes. It can lay claim to being at least the basis for an objective interpretation, and it can be argued that in taking propensities to be real properties of experimental arrangements it constitutes a kind of realistic interpretation. But let me conclude with a cautionary note. When Popper claims to provide a realistic interpretation of quantum mechanics, he is tacitly asserting his allegiance with a long, continuing philosophical tradition, and though he never, to my knowledge, explicitly mentions it, one important assumption of traditional realism is the mutual independence of observer and observed. Yet precisely this assumption is put in question by quantum mechanics. In Bohr's words:

...the elucidation of the paradoxes of atomic physics has disclosed the fact that the unavoidable interaction between the objects and the measuring instruments sets an absolute limit to the possibility of speaking of a behaviour 
of atomic objects which is independent of the means of observation. [32, p. 25]

If one could assert the simultaneous definiteness of the properties of interacting systems, then the independence of observer and observed could be maintained, for they could then each be assigned a separate state. So Popper's version of the propensity interpretation, incorporating the definiteness thesis, qualifies as a realistic interpretation in the traditional sense. But if definiteness is denied, as I think it ought to be, and the holism of the orthodox quantum mechanical description of interactions is accepted, then it must be understood that, on its own, the propensity interpretation's place in the tradition is open to question.

\section{Author's Note}

This paper was originally written in 1981 by invitation for inclusion in a Popper Festschrift. But for reasons not worth recalling now, it was not published in that volume. It is presented here with only minimal editorial changes to update some tenses, reflecting the fact of Popper's since having died and, in only one case, to call attention to one of the author's subsequent papers. These circumstances explain the somewhat dated quality that some readers might note.

\section{References}

[1] Popper KR. Quantum Mechanics without 'The Observer'. In: Quantum Theory and Reality, Bunge M (editor), New York: Springer, 1967, pp. 7-43.

[2] Feyerabend PK. On a recent critique of complementarity: part I. Philosophy of Science 1968; 35 (4): 309-331.

[3] Feyerabend PK. On a recent critique of complementarity: part II. Philosophy of Science 1969; 36 (1): 82-105.

[4] Heisenberg W. Über den anschaulichen Inhalt der quantentheoretischen Kinematik und Mechanik. Zeitschrift für Physik A 1927; 43 (3-4): 172-198.

[5] Bohr N. The quantum postulate and the recent development of atomic theory. Nature 1928; 121 (3050): 580-590.

[6] Bohr N. The Quantum Postulate and the Recent Development of Atomic Theory. In: Atti del Congresso Internazionale dei Fisici, Como, 11-20 Settembre 1927 (Bologna: Zanichelli, 1928), vol. 2, pp. 565588 .
[7] Bohr N. The Quantum Postulate and the Recent Development of Atomic Theory. In: Atomic Theory and the Description of Nature, Cambridge: Cambridge University Press, 1934, pp. 52-91.

[8] Jammer M. The Philosophy of Quantum Mechanics: The Interpretations of Quantum Mechanics in Historical Perspective. New York: John Wiley \& Sons, 1974.

[9] Bohr N. Can quantum-mechanical description of physical reality be considered complete? Physical Review 1935; 48 (8): 696-702. http://dx.doi, org/10.1103/PhysRev.48.696

[10] Bohr N. Discussion with Einstein on Epistemological Problems in Atomic Physics. In: Albert Einstein: Philosopher-Scientist, Schilpp PA (editor), The Library of Living Philosophers, vol. 7, LaSalle, Illinois: Open Court, 1949, pp. 201-241.

[11] Howard D. Who invented the "Copenhagen Interpretation"? A study in mythology. Philosophy of Science 2004; 71 (5): 669-682. http://dx.doi, org/10.1086/425941

[12] Heisenberg W. The development of the interpretation of quantum theory. In: Niels Bohr and the Development of Physics, Pauli W (editor), Condon: Pergamon, 1955, pp. 12-29.

[13] Popper KR. The Logic of Scientific Discovery. London, New York: Routledge, 2002.

[14] Popper KR. Intellectual Autobiography. In: The Philosophy of Karl Popper, Schilpp PA (editor), The Library of Living Philosophers, vol. 14, LaSalle, Illinois: Open Court, 1974.

[15] Popper KR. Unended Quest: An Intellectual Autobiography. London, New York: Routledge, 2002.

[16] Schrödinger E. Collected Papers on Wave Mechanics. Shearer JF, Deans WM (translators), London: Blackie \& Son, 1928.

[17] Scott WT. Erwin Schrödinger: An Introduction to His Writings. Amherst, Massachusetts: University of Massachusetts Press, 1967.

[18] Born M. Zur Quantenmechanik der Stoßvorgänge. Zeitschrift für Physik A 1926; 37 (12): 863-867. http://dx.doi.org/10.1007/BF01397477 
[19] Popper KR. Indeterminism in quantum physics and in classical physics: part I. The British Journal for the Philosophy of Science 1950; 1 (2): 117-133. http://dx.doi.org/10.1093/bjps/I.2.117

[20] Popper KR. Indeterminism in quantum physics and in classical physics: part II. The British Journal for the Philosophy of Science 1950; 1 (3): 173-195. http://dx.doi.org/10.1093/bjps/I.3.173

[21] Einstein A. Letter 84, 3 March 1947. In: The Born-Einstein letters: correspondence between Albert Einstein and Max and Hedwig Born from 1916-1955, with commentaries by Max Born. London: Macmillan, 1971. http://archive.org/ details/TheBornEinsteinLetters

[22] Heisenberg W. The Physical Principles of the Quantum Theory. Eckart C, Hoyt FC (translators), New York: Dover, 1949.

[23] von Neumann J. Mathematical Foundations of Quantum Mechanics. Beyer RT (translator), Princeton: Princeton University Press, 1955.

[24] Bell JS. On the problem of hidden variables in quantum mechanics. Reviews of Modern Physics 1966; 38 (3): 447-452.http://dx.doi.org/10.1103/ RevModPhys.38.447

[25] Popper KR. Probability magic or knowledge out of ignorance. Dialectica 1957; 11 (34): 354-374. http://dx.doi.org/10.1111/j 1746-8361.1957.tb01643.x

[26] Schlick M. Die Kausalität in der gegenwärtigen Physik. Naturwissenschaften 1931; 19 (7): 145-162. http://dx.doi.org/10.1007/BF01516406

[27] Jeans JH. The New Background of Science. New York: Macmillan, 1933. http://archive.org/ details/newbackgroundofs006677mbp

[28] Heisenberg W. The representation of nature in contemporary physics. Daedalus 1958; 87 (3): 95-108.

[29] Popper KR. Objective Knowledge: An Evolutionary Approach. New York: Oxford University Press, 1972.

[30] d'Espagnat B. Conceptual Foundations of Quantum Mechanics. Reading, Massachusetts: Perseus Books, 1999.

[31] Wigner EP. Remarks on the mind-body question. In: The Scientist Speculates, Good IJ (editor), London: Heinemann, 1961, pp.284-302.
[32] Bohr N. Atomic Physics and Human Knowledge. New York: John Wiley \& Sons, 1958. http://archive.org/details/ AtomicPhysicsHumanKnowledge

[33] Bohr N. The causality problem in atomic physics. In: Niels Bohr Collected Works, vol.7. Foundations of Quantum Physics II (1933-1958), Kalckar J (editor), Elsevier, 1996, pp.299-322.http://dx.doi.org/ 10.1016/s1876-0503(08)70376-1

[34] Bohr N. Quantum physics and philosophy - causality and complementarity. In: Niels Bohr Collected Works, vol.7. Foundations of Quantum Physics II (1933-1958), Kalckar J (editor), Elsevier, 1996, pp.385-394. http://dx.doi.org/10. 1016/s1876-0503(08)70381-5

[35] Heisenberg W. Physics and Philosophy: The Revolution in Modern Science. Unwin University Books, London: George Allen \& Unwin, 1971. http://www.archive.org/details/ PhysicsPhilosophy

[36] Carnap R. Logical Foundations of Probability. London: Routledge and Kegan Paul, 1950.

[37] Popper KR. The propensity interpretation of probability. The British Journal for the Philosophy of Science 1959; 10 (37): 25-42. http://dx.doi. org/10.1093/bjps/X.37.25

[38] Bohr N. On the notions of causality and complementarity. Dialectica 1948; 2 (3-4): 312-319. http://dx.doi.org/10.1111/j.1746-8361. 1948.tb00703.x

[39] Bohr N. On the notions of causality and complementarity. Science 1950; 111 (2873): 51-54. http:// dx.doi.org/10.1126/science.111.2873.51

[40] Bohr N. The Unity of Human Knowledge. In: Niels Bohr Collected Works, vol.10. Complementarity Beyond Physics (1928-1962), David F (editor), Elsevier, 1999, pp.155-160. http://dx.doi.org/ 10.1016/s1876-0503(08)70208-1

[41] Bohr N. The Rutherford Memorial Lecture 1958: Reminiscences of the Founder of Nuclear Science and of Some Developments Based on his Work. Proceedings of the Physical Society 1961; 78 (6): 1083-1115. http://dx.doi.org/10.1088/ 0370-1328/78/6/301 
[42] Hooker CA. The nature of quantum mechanical reality: Einstein versus Bohr. In: Paradigms \& Paradoxes: The Philosophical Challenge of the Quantum Domain, Colodny RG (editor), University of Pittsburgh Series in the Philosophy of Science, vol. 5, Pittsburgh: University of Pittsburgh Press, 1972, pp. 67-302.

[43] Popper KR. Zur Kritik der Ungenauigkeitsrelationen. Naturwissenschaften 1934; 22 (48): 807-808. http://dx.doi.org/10.1007/BF01496543

[44] Einstein A, Podolsky B, Rosen N. Can quantummechanical description of physical reality be considered complete? Physical Review 1935; 47 (10): 777-780. http://dx.doi.org/10.1103/ PhysRev.47.777

[45] Bohm D. Quantum Theory. New York: Dover Publications, 1989.

[46] Furry WH. Note on the quantum-mechanical theory of measurement. Physical Review 1936; 49 (5): 393-399. http://dx.doi.org/10.1103/ PhysRev.49.393

[47] Howard D. Complementarity and Ontology: Niels Bohr and The Problem of Scientific Realism in Quantum Physics. Ph. D. Dissertation, Boston University, 1979.

[48] Clauser JF, Shimony A. Bell's theorem. Experimental tests and implications. Reports on Progress in Physics 1978; 41 (12): 1881-1927. http://dx. doi.org/10.1088/0034-4885/41/12/002
[49] Mermin ND. Quantum mysteries for anyone. The Journal of Philosophy 1981; 78 (7): 397-408. http: //www.jstor.org/stable/2026482

[50] Belinfante FJ. A Survey of Hidden Variable Theories. Monographs in Natural Philosophy, New York: Pergamon, 1973.

[51] Shimony A. Metaphysical problems in the foundations of quantum mechanics. International Philosophical Quarterly 1978; 18 (1): 3-17.

[52] d'Espagnat B. The quantum theory and reality. Scientific American 1979; 241 (11): 158-181.

[53] Popper KR. The propensity interpretation of the calculus of probability and the quantum theory. In: Observation and Interpretation: A Symposium of Philosophers and Physicists. Proceedings of the Ninth Symposium of the Colston Research Society held in the University of Bristol, April 1st-April 4th, 1957, Körner S (editor), London: Butterworth Scientific Publications, 1957, pp.65-70.

[54] Sneed JD. Review of "Quantum Theory and Reality" editted by Mario Bunge. Synthese 1968; 18 (4): 464-467. http://dx.doi.org/10.1007/ BF00484982

[55] Suppes P. Popper's analysis of probability in quantum mechanics. In: The Philosophy of Karl Popper, vol.2, Schilpp PA (editor), LaSalle, Illinois: Open Court Publishing Company, 1974, pp.760-774. 